\title{
Haem oxygenase-1 expression is diminished in alveolar macrophages of patients with COPD
}

To the Editor:

We read with great interest the paper by MAESTRELLI et al. [1] recently published in the European Respiratory Journal $(E R J)$. They showed a lower expression of haem oxygenase-1 (HO-1) in alveolar macrophages in lung tissue samples of patients with chronic obstructive pulmonary disease (COPD) than of controls. Unfortunately, the authors included a mixed group of smokers and nonsmokers. This makes their results more difficult to interpret since cigarette smoke is known to influence HO-1 expression as shown in an earlier paper by the same authors [2]. Furthermore, the lung tissue samples in their COPD patient group were mainly derived by lung volume resection surgery and in the control group from resection because of lung cancer [1]. We have recently analysed bronchoalveolar lavage (BAL) fluid of exsmokers with COPD and compared these to healthy exsmokers thereby ruling out the effects of current smoking history.

Thirteen patients with COPD, according to American Thoracic Society (ATS) criteria, were included with a mean age of $64 \mathrm{yrs}$, forced expiratory volume in one second (FEV1) $59 \%$ predicted, reversibility $6.0 \%$ of pred FEV1, and 26 packyrs of smoking. Eleven age matched control subjects were recruited, with a mean age of 58 yrs, FEV1 $103 \%$ pred, reversibility $3.4 \%$ of pred FEV1, and 25 pack-yrs [3]. All subjects had no history of asthma or atopy and had a negative skin test for 18 common aeroallergens. Treatment with inhaled corticosteroids was discontinued at least 1 month prior to the study and no treatment with oral corticosteroids and antibiotics was allowed in the same period. The airways were lavaged, using the first $50 \mathrm{~mL}$ for bronchial wash and the subsequent $150 \mathrm{~mL}$ for bronchoalveolar lavage as described previously [3]. The lavage alveolar macrophage count was comparable in both groups. Immunostaining of cytospins was performed with a monoclonal antibody against HO-1 (Beckton Dickinson, Franklin Lakes, NJ, USA). Total HO-1 expression on macrophages was scored semi-quantitatively in a blinded panel by two independent observers; scores ranged from 0 (negative), 1, 2, to 3 (maximal) according to increasing intensity. Four hundred alveolar macrophages were counted on two slides and the HO-1 scores were added up. The overall HO-1 expression of BAL alveolar macrophages was significantly lower in the exsmoking COPD patients when compared to the healthy exsmoking controls (fig. 1).

The lower expression of haem oxygenase-1 in lavage macrophages observed in our patients with moderate-to-severe chronic obstructive pulmonary disease (Global initiative for Chronic Obstructive Lung Disease stage 2-3, [4]) corroborates the findings of MAEsTRelli et al. [1] on surgical specimens. It extends their findings in that this decreased expression is also present in patients and controls without current smoking, i.e. smoking does not directly influence the haem oxygenase-1 expression. Haem oxygenase-1 has very potent anti-oxidative and anti-inflammatory capacities, for instance protection against allograft rejection and hyperbaric oxygen injury in animal models [5]. The results of MAESTRELLI et al. [1] and our own results are compatible with the hypothesis that haem oxygenase- 1 is insufficiently upregulated in chronic obstructive pulmonary disease. This might be due to genetic polymorphisms: YAMADA et al. [6] for instance have shown a larger number of (GT)n repeats in the haem oxygenase-1 gene promoter to decrease inducibility of

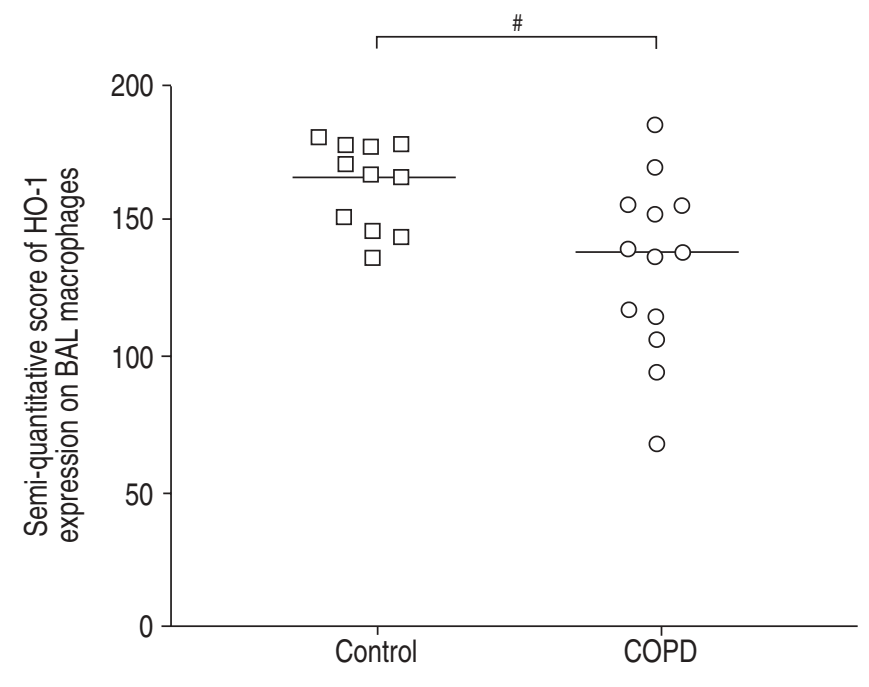

Fig. 1. - Total haem oxygenase-1 (HO-1) expression. Semi-quantitative total score of HO-1 expression on alveolar macrophages in bronchoalveolar lavage fluid for exsmoking chronic obstructive pulmonary disease patients $(\bigcirc)$ and age-matched healthy exsmoking controls ( $\square$ ). The horizontal line represents the median. ${ }^{\#}$ : $\mathrm{p}=0.018$.

haem oxygenase-1 by reactive oxygen species. We believe that further research to elucidate the exact role of haem oxygenase-1, its genetic variability and its downstream products in chronic obstructive pulmonary disease is warranted.

D-J. Slebos*\# H.A.M. Kerstjens*, S.R. Rutgers*, H.F. Kauffman ${ }^{\#}$, A.M.K. Choi ${ }^{*}$, D.S. Postma*

*Depts of Pulmonary diseases, and ${ }^{\#}$ Allergology, University Hospital Groningen, the Netherlands, and Division of Pulmonary, Allergy and Critical Care Medicine, University of Pittsburgh School of Medicine, Pittsburgh, PA, USA.

\section{References}

1. Maestrelli P, Paska C, Saetta M, et al. Decreased haem oxygenase-1 and increased inducible nitric oxide synthase in the lung of severe COPD patients. Eur Respir $J$ 2003; 21 : 971-976.

2. Maestrelli P, El Messlemani AH, De Fina O, et al. Increased Expression of Heme Oxygenase (HO)-1 in Alveolar Spaces and HO-2 in Alveolar Walls of Smokers. Am J Respir Crit Care Med 2001; 164: 1508-1513.

3. Rutgers SR, Timens W, Kaufmann HF, van der Mark TW, Koeter GH, Postma DS. Comparison of induced sputum with bronchial wash, bronchoalveolar lavage and bronchial biopsies in COPD. Eur Respir J 2000; 15: 109-115.

4. Fabbri LM, Hurd SS, GOLD Scientific Committee. Global Strategy for the Diagnosis, Management and Prevention of COPD: 2003 update. Eur Respir J 2003; 22: 1-2.

5. Slebos DJ, Ryter SW, Choi AM. Heme oxygenase-1 and carbon monoxide in pulmonary medicine. Respir Res 2003; 4: 7.

6. Yamada N, Yamaya M, Okinaga S, et al. Microsatellite 
polymorphism in the heme oxygenase-1 gene promoter is associated with susceptibility to emphysema. Am J Hum Genet 2000; 66: 187-195.

\section{From the authors:}

We thank D-J. Slebos and colleagues for their comments relating to our paper on the imbalance between haem oxygenase (HO)-1 and inducible nitric oxide synthase (iNOS) in the lungs of severe chronic obstructive pulmonary disease (COPD) patients [1].

Our data showed that expression of HO-1 was decreased in alveolar macrophages in severe COPD, whereas iNOS expression in type- 2 pneumocytes was increased. It is of great interest to see that the results provided by Slebos and colleagues confirm the part of our findings related to HO-1 expression in alveolar macrophages. Since the origin of macrophages retrieved by bronchoalveolar lavage is undetermined, the findings of Slebos and colleagues might indicate that the altered expression of HO-1 in COPD is not limited to the alveolar compartment, but extends to macrophages from the bronchial lumen.

We believe that the presence of current smokers among our patients was a negligible confounding factor for the different expression of HO-1 in severe COPD as compared with control smokers without impairment of lung function [1]. In fact, it was shown in our previous paper [2] that upregulation of HO1 in alveolar macrophages of subjects with smoking history was irrespective of the fact they were current or ex-smokers. The data provided by Slebos and colleagues support the interpretation that HO-1 expression is not influenced directly by cigarette smoke.

We are aware of the potential bias in studies performed on surgically resected specimens, due to the presence of cancer itself or to the selection of the worst part of the lungs in patients who underwent lung volume reduction surgery. However, surgical specimens are precious tissue because they allow direct examination of lung pathology in living patients, which would be otherwise impossible for obvious ethical reasons. This opportunity is particularly relevant in COPD, as peripheral lung is the site where most of the pathological processes take place. This compartment of the lungs cannot be explored using bronchial biopsies. The alternative would be performing post-mortem studies, but in this case clinical and functional characterisation of the subjects is problematic.

One real limitation of immunohistological studies is that they are not suitable for quantitative evaluation of an enzyme such as HO-1. We assumed that the number of positively stained cells is related to enzyme activity, but this may not be true. The attempt to express the results of immunostaining as a score of intensity does not appear to overcome such limitation unless this score is validated by other measurements.

\section{P. Maestrelli}

Dept of Environmental Medicine and Public Health, University of Padova, Padova, Italy

\section{References}

1. Maestrelli P, Paska C, Saetta M, et al. Decreased haem oxygenase-1 and increased inducible nitric oxide synthase in the lung of severe COPD patients. Eur Respir $J$ 2003; 21: 971-976.

2. Maestrelli P, El Messlemani AH, De Fina O, et al. Increased expression of heme oxygenase (HO)-1 in alveolar spaces and HO-2 in alveolar walls of smokers. Am J Respir Crit Care Med 2001; 164: 1508-1513.

\section{Diagnostic implications of telomerase activity in pleural effusions}

\section{To the Editor:}

In a recent issue of the European Respiratory Journal, DIKMEN et al. [1] analysed the diagnostic efficacy of telomerase activity in pleural fluid for distinguishing between benign and malignant effusions. They found that the sensitivity and specificity of telomerase activity for diagnosing malignancy was 82 and $80 \%$, respectively. With these data the authors concluded that telomerase activity is a highly sensitive diagnostic biomarker for malignancy and may be used as an adjunct to cytological findings in determining malignant pleural effusions. However, in our opinion, this affirmation can not be applied to clinical practice.

Sensitivity and specificity are important points in the study of the diagnostic value of a test. However, from a clinical point of view, other measures of diagnostic accuracy are of great interest [2], i.e. likelihood ratios and predictive values. Likelihood ratios are the likelihood that a given test result would be expected in a patient with the target disorder compared to the likelihood that the same result would be expected in a patient without the target disorder, and predictive values are the post-test probability $[3,4]$. We calculated these measures using the data reported by DIKMEN et al. [1], and obtained positive and negative likelihood ratios of 4.1 and 0.22 , respectively, which are of modest diagnostic interest [3, 4]. The positive predictive value, in this case, represents the probability that a patient has a malignant pleural effusion if telomerase activity is detected in the pleural fluid. Although it obviously depends on pre-test probability, this predictive value is very low, especially when the prevalence (pre-test probability) is $\sim 20-30 \%$, as is usually observed in general hospitals (table 1) [5]. These findings are similar to those obtained when using classical tumour markers [5].

In our experience [6], the determination of C-reactive protein (CRP) in pleural exudates, which is an inexpensive test and easy to perform, would be of greater interest. A low CRP level $\left(<20 \mathrm{mg} \cdot \mathrm{L}^{-1}\right)$ has a better positive likelihood ratio and positive predictive value for diagnosing malignancy than those of telomerase activity, with the additional advantage that a level $>45 \mathrm{mg} \cdot \mathrm{L}^{-1}$ virtually rules out this possibility.

In conclusion, we consider that the measurement of telomerase activity in pleural fluid adds little to the diagnostic 